\title{
Tailoring swelling to control softening mechanisms during cyclic loading of PEG/cellulose hydrogel composites
}

\author{
A. Khoushabi ${ }^{\mathrm{a}, \mathrm{b}}$, C.S. Wyss ${ }^{\mathrm{a}, \mathrm{b}}$, B. Caglar ${ }^{\mathrm{a}}$, D. Pioletti ${ }^{\mathrm{b}}$, P.-E. Bourban ${ }^{\mathrm{a}, *}$ \\ a Laboratory for Processing of Advanced Composite (LPAC), Ecole Polytechnique Fédérale de Lausanne (EPFL), CH-1015, Lausanne, Switzerland \\ ${ }^{\mathrm{b}}$ Laboratory of Biomechanical Orthopedics (LBO), Ecole Polytechnique Fédérale de Lausanne (EPFL), CH-1015, Lausanne, Switzerland
}

\section{A B S T R A C T}

One of the novel approaches for discogenic lower back pain treatment is to permanently replace the core of the intervertebral disc, so-called Nucleus Pulposus, through minimally invasive surgery. Recently, we have proposed Poly(Ethylene Glycol) Dimethacrylate (PEGDM) hydrogel reinforced with Nano-Fibrillated Cellulose (NFC) fibers as an appropriate replacement material. In addition to the tuneable properties, that mimic those of the native tissue, the surgeon can directly inject it into the degenerated disc and cure it in situ via UV-light irradiation. However, in view of clinical applications, the reliability of the proposed material has to be tested under long-term fatigue loading. To that end, the present study focused on the characterization of the fatigue behavior of the composite hydrogel and investigated the governing physical phenomena behind it. The results show that composite PEGDM-NFC hydrogel withstands the 10 million compression cycles at physiological condition. However, its modulus decreases by almost $10 \%$ in the first cycle and then remains constant, while cyclic loading does not affect the neat PEGDM hydrogel. The observed softening behavior has similar characteristics of the Mullins effect. It is shown that the reduction of modulus is due to the gradual change of NFC network, which is highly stretched in the swollen state. Moreover, the swelling degree of the matrix is correlated to the extent of softening during cyclic loading. Consequently, softening can be minimized by lowering the swelling of the composite hydrogel.

\section{Introduction}

Hydrogels have increasingly gained importance in the biomedical field, in particular, for tissue engineering such as muscle or cartilage repair, drug delivery systems, implants and wound dressing [1-3]. The high water content of biocompatible hydrogels is suitable for cell encapsulation and nutriment transport. In addition, a wide range of properties can be obtained and tuned according to the target application requirements $[4,5]$.

From the surgical point of view employing hydrogels is beneficial since their liquid precursor can be injected through a minimally invasive surgery and cured in situ via different stimuli such as heat or chemical reaction [6]. In this context, using photopolymerization as a curing method has an advantage over others since it provides the surgeon with more temporal and spatial control over the curing reaction $[7,8]$. However, the low mechanical properties of current hydrogels limit their application in this context.

Designing composite hydrogel is one of the approaches proposed to improve their mechanical properties [9-12] The hydrogel properties can be tailored by changing the reinforcement concentration while preserving in situ applicability of the hydrogel implants [13]. Furthermore, hybrid and hierarchical microstructure of various natural tissues such as cartilage or the core of intervertebral disc (i.e. nucleus pulpous [12]) motivates a nature-inspired approach to design composite implant materials to replace the native tissues [14-17].

In previous works, the authors have reported development of photopolymerizable hydrogel systems reinforced with Nano-Fibrillated Cellulose (NFC) fibers for permanent load-bearing implant applications [7]. Cellulose fibers have been chosen as reinforcement fibers due to their large surface-to-volume ratio, high stiffness and strength $[12,18]$ as well as their biocompatibility $[19,20]$ and biodurability [21]. It was demonstrated that the addition of NFC reinforcements improves the hydrogel stiffness without compromising the failure strength or significant reduction of the water content. Moreover, the incorporated NFC fibers were shown to serve as a light-scattering source when the precursor solution is illuminated by UV light [22]. The latter accelerates the kinetics of photopolymerization and consequently allows reducing the required concentration of photoinitiator. The applicability and reliability of such an approach in design of hydrogels as orthopedic implant materials is confirmed using an ex vivo bovine organ model [7].

The physiological loads applied to biomedical components are mostly cyclic and a long-term service life is often expected from permanent implants. Hence, fatigue life assessment is of substantial importance in the evaluation of a biomechanical applicability of the proposed NFC reinforced hydrogels.

In previous work we have investigated the in situ response of the composite hydrogel under fatigue loading employing ex vivo animal model [7]. The ex vivo experiments indicate that the repaired disc is

\footnotetext{
* Corresponding author.

E-mail address: pierre-etienne.bourban@epfl.ch (P.-E. Bourban).
} 


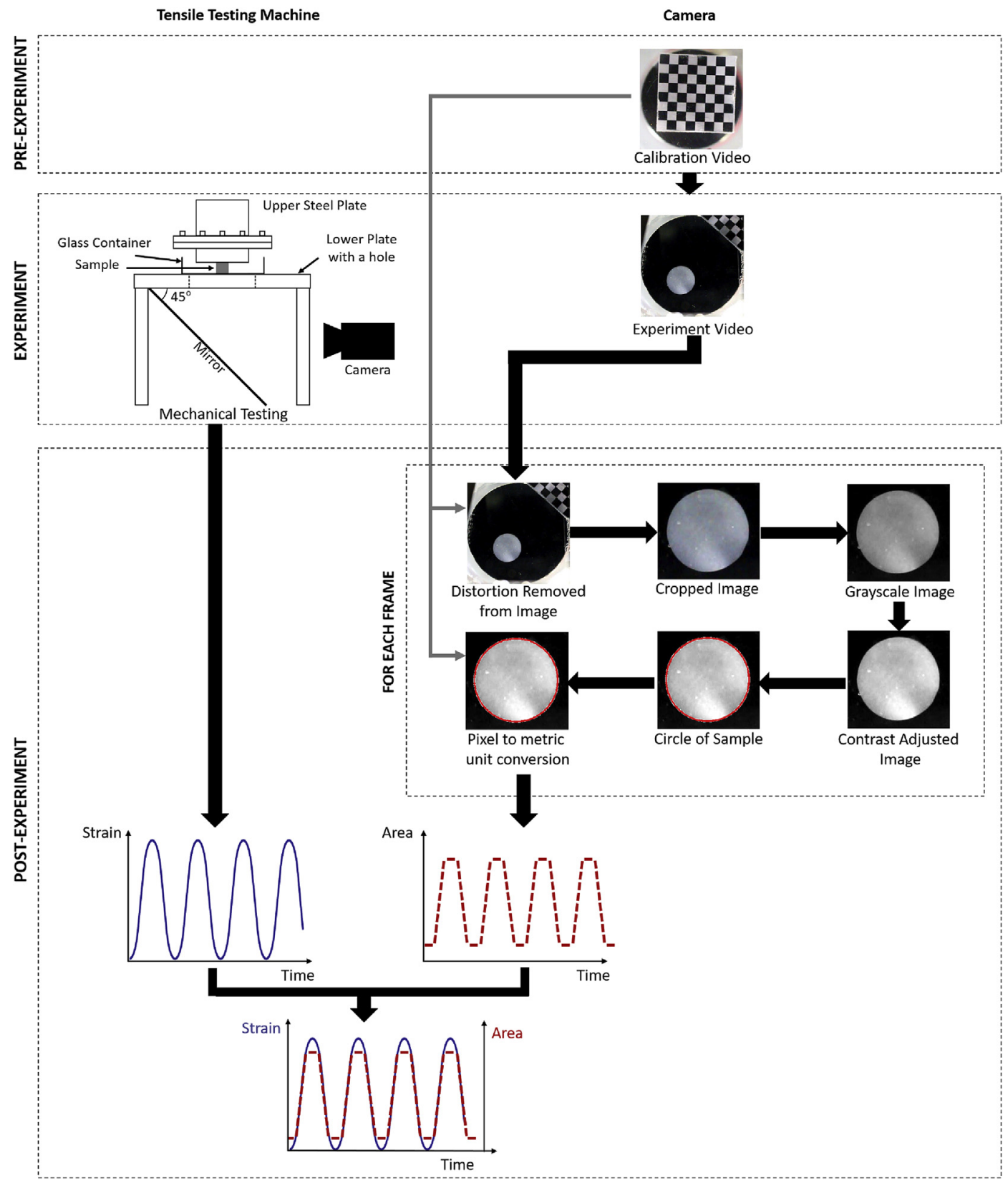

Fig. 1. Procedure adopted for simultaneous area measurement during the fatigue test.

able to withstand cyclic loading and maintain its height over 0.5 million cycles without being damaged. Histological analysis of the repaired disc after the cyclic loading revealed a continuous and intact tissue/implant interface. Although such testing method is robust and yields realistic results regarding the applicability of the implant materials under cyclic loading, its duration as well as applied loading amplitude are constrained by tissue degeneration in the testing conditions [23]. Moreover, in the ex vivo model only the confined and unswollen state of the composite hydrogel could be evaluated. Yet, an implant material can undergo millions of loading cycles [24] that requires a thorough fatigue characterization of NFC reinforced hydrogels beyond the limitations of the ex vivo animal model testing.

A wealth of literature exists on fatigue of soft materials such as hydrogels $[25,26]$. Nevertheless, fatigue studies on composite hydrogels are very limited and in particular only few works have been reported on composite hydrogels reinforced with cellulose fibers [27]. In this work, long-term mechanical reliability of the composite hydrogels based on PEGDM hydrogel matrix and NFC reinforcements is assessed in the loading condition similar to the one of the human intervertebral disc (maximum 20\% strain). Fatigue characterizations are performed under both low cycle and high cycle loadings up to 10 million cycles. The present mechanistic study aims to evaluate the loading response mechanism of the hybrid microstructure of the NFC reinforced hydrogel to cyclic loadings. The main focus is given to the identification of the governing physical phenomenon behind the fatigue behavior of NFCreinforced hydrogel.

\section{Materials and methods}

\subsection{Materials}

Poly(ethylene glycol) dimethacrylate (PEGDM), with the molecular weight of $20 \mathrm{kDa}$ was synthesized as previously described [28]. Nanofibrillated cellulose (NFC) was prepared by fibrillation of cellulose pulp (bleached softwood pulp, Elemental Chlorine Free (ECF), Zellstoff Stendal, Germany) using a high-shear homogenizer, by pumping the 
suspension through two consecutive chambers of 400 and $200 \mu \mathrm{m}$ for 12 passes. As reported [29], the hemicellulose and cellulose contents are of 12.6 and $81.3 \%$ respectively while the diameters of NFC are in the range of $2-100 \mathrm{~nm}$ and their lengths are in a few micrometers range as observed by SEM.

\subsection{Hydrogels synthesis}

Hydrogel precursors were prepared according to an established protocol [13]. PEGDM, phosphate buffered saline (PBS, pH 7.4, Gibco), NFC and $0.1 \mathrm{~g} \mathrm{ml}^{-1} \%$ of Irgacure 2959 (BASF), were mixed as photoinitiator using ultra-turrax (IKA T25 digital, SN 25 10G). The homogenized suspension was degassed at 20 mbar and casted in cylindrical polypropylene mold ( $\varnothing 8 \mathrm{~mm} \times 4 \mathrm{~mm}$ ). The molds were covered with microscope slides and cured for $30 \mathrm{~min}$ under UV-light irradiation with monochromatic $365 \mathrm{~nm}$ ultra violet lamp (AxonLab) with an intensity of $5 \mathrm{~mW} \mathrm{~cm}^{-2}$.

If not stated otherwise the neat and composite hydrogel correspond to $10 \mathrm{wt}$.\% PEGDM $20 \mathrm{kDa}$ hydrogel without NFC and with 0.5 vol.\% NFC respectively.

\subsection{Swelling ratio and water content measurement}

Hydrogels volume (V) were measured using a density determination kit before and after mechanical tests and the swelling ratios were calculated via: Swelling ratio (\%) $=V_{\text {swollen hydrogel }}-V_{\text {as synthesised hydrogel }} / V_{\text {as synthesised hydrogel }}$.

\subsection{Mechanical testing}

\subsubsection{Mechanical test set-up}

The mechanical response of the hydrogels in uniaxial unconfined compression were evaluated using an Instron E3000 linear mechanical testing machine (Norwood, MA, USA) equipped with a $250 \mathrm{~N}$ load cell. The hydrogel cross-section were measured during the test with the aid of a camera and the true stress values $(\sigma)$ were directly calculated from: $\sigma=\mathrm{F}$ / $\mathrm{A}$, where $\mathrm{F}$ is the recorded applied load at each time point and $\mathrm{A}$ is the corresponding area of the hydrogel sample recorded by a video camera.

As schematically illustrated in Fig. 1 three stages were considered in order to obtain the hydrogels area during the test: (i) pre-experiment, (ii) experiment, and (iii) post-experiment. In pre-experiment stage, a calibration video is recorded which is used for removing the distortion and finding the correspondence of pixel size to metric size (see Fig. 1pre-experiment box).

The mechanical testing experiment was done using a set-up shown in Fig. 1- experiment box, where a transparent case allows simultaneous recording of the hydrogel sample with the aid of installed camera. To this end, a Canon EOS 700D DSLR camera equipped with $18-55 \mathrm{~mm}$ lens is placed facing the mirror to ensure that the sample stays within the field of view of the camera throughout the experiment. Experiment is recorded at a resolution of $1280 \times 720$ pixels and $60 \mathrm{fps}$.

In post-experiment stage, frames of video recorded during the experiment were imported to MATLAB and corrected for lens distortion (pincushion, barreling). In the next step, frames were cropped to minimize the computational load. Cropped images were converted to grayscale images using the built-in rgb2gray function in MATLAB. The intensity of the grayscale images was adjusted using the built-in imadjust function. This step stretches the intensity map of the grayscale image in order to ease the isolation of the sample from the rest of the image. Then, circle of the sample was estimated using the built-in circle finding function, namely imfindcircles function. Corresponding area is then converted from pixels to metric units using the pixel correspondence obtained in the pre-experiment stage. Once the area of the sample is calculated for all the frames, the two signals, namely the applied strain signal from the instron machine and the area signal obtained from the video were aligned using the built-in $x$ corr function (see Fig. 1 - post-experiment box). This cross-correlation step is required to match the origins of the time scales of the two signals, which are originally shifted with respect to each other since video recording and mechanical test are not started at the same instant.

\subsubsection{Mechanical testing parameters}

The mechanical responses of hydrogels were evaluated at different states namely: "unswollen" or "as-prepared" i.e. after synthesis, "swollen" i.e. immersed in PBS for one week at room temperature, "after relaxation" i.e. immersed in PBS for 1 week after mechanical test and "after rehydration " i.e. dried in vacuum oven at $40{ }^{\circ} \mathrm{C}$ for 3 days after mechanical test followed by immersion in PBS for a week at room temperature. Minimum 3 replicates have been tested for each experiment condition. Swollen hydrogels were immersed in PBS during mechanical test, whereas unswollen hydrogels were tested in dry chamber. Tests were performed at room temperature.

In the long-term fatigue test a uniaxial compressive strain of $20 \%$ was applied to swollen neat and composite hydrogels for respectively $10^{6}$ and $10^{7}$ cycles. As confirmed by preliminary tests, the composite hydrogel response is not rate dependence in unconfined condition. Thus the frequency of $10 \mathrm{~Hz}$ is chosen to expedite the fatigue test. In the progressive strain experiment, the hydrogel sample was compressed to various upper limits of applied strain. The upper limits start from 10\% and goes up to $70 \%$ by an increment of $10 \%$. 10 cycles of compression were applied for each upper strain limit. All the cyclic loadings were applied as a sinusoidal wave. If not specified otherwise compressions were applied at the rate of $1 \mathrm{~mm} / \mathrm{s}$.

\subsubsection{Experimental data analysis}

The elastic modulus (E) were calculated by linear interpolation of the true stress-strain curve between the strains of 0.05-0.1. The elastic modulus reported from progressive strain experiment are measured from the true strain-stress curve of the 10th loading cycle at each applied strain level. The energy dissipation is calculated as a surface between the loading/unloading true stress-strain curves. The softening of the hydrogel is reported as:

Softening $(\%)=100\left(E_{\text {virgin }}-E_{10}\right.$ th cycle at each applied strain level $) / E$ virgin

where $E_{\text {virgin }}$ is the elastic modulus of the hydrogel sample measured from the first loading cycle.

\subsubsection{Fluorescence confocal microscopy}

The morphology of the NFC fibres embedded in PEGDM hydrogel matrix were observed via fluorescent confocal microscopy with the $20 \mathrm{x}$ lens and the laser of $405 \mathrm{~nm}$. In order to distinguish NFC fibres from the matrix, the composite hydrogel was dyed with Calcofluor White stain (Sigma-Aldrich, Buchs, $\mathrm{CH}$ ).

Supplementary video related to this article can be found at https:// doi.org/10.1016/j.compscitech.2018.08.043.

\section{Results and discussion}

\subsection{High-cycle fatigue at equilibrium swelling}

The evolution of the hydrogels' elastic modulus during the cyclic deformation is shown in Fig. 2. The results indicate the long-term loadbearing reliability of the composite hydrogel up to 10 million cycles. However, while the modulus of the neat hydrogel is not affected by cyclic loading up to 1 million cycles, the composite hydrogel modulus decreases by almost $10 \%$ in the first few cycles and then remains constant at a higher value than for the neat hydrogel.

Since the stiffness reduction in the composite hydrogel only occurs in the first 10 cycles, a low cycle fatigue approach is adopted for further characterization. The cyclic loading response of the neat hydrogel is also investigated throughout the work as a reference material representing the matrix of the composite hydrogel. 


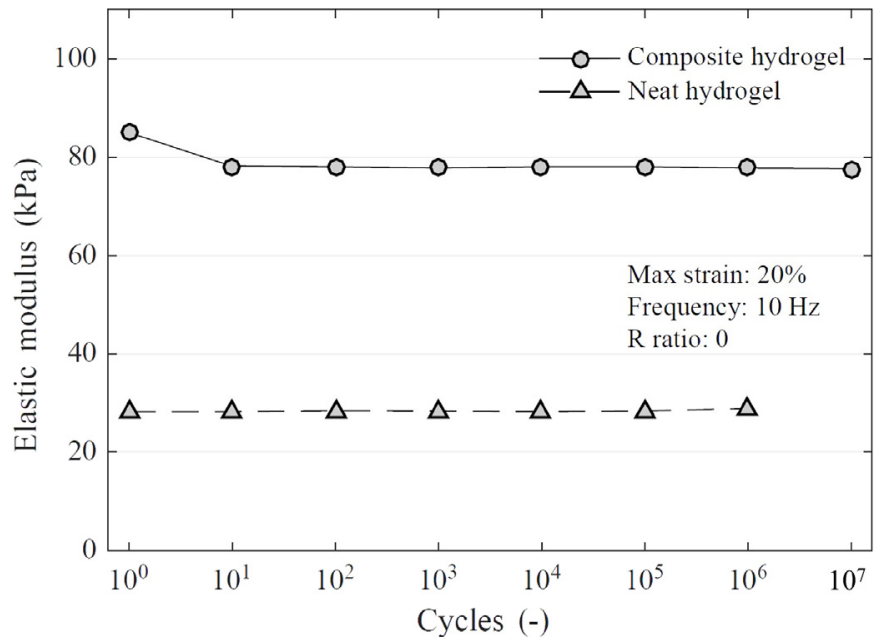

Fig. 2. The evolution of elastic modulus of the swollen neat and swollen composite hydrogel under fatigue load.

\subsection{Low-cycle fatigue at equilibrium swelling}

Representative cyclic stress-strain curves of the neat and composite hydrogels are shown in Fig. 3-a. The loading/unloading curves of the neat hydrogel at strain amplitude of $70 \%$ follows almost the same path indicating no significant change in the deformation mechanism by increasing the deformation cycles. The elastic modulus and dissipated energy in cyclic loading of the neat hydrogel remains almost identical during the successive loading cycles (see Fig. 3-b and c). However, the dissipated energy in the composite hydrogel decreases significantly in

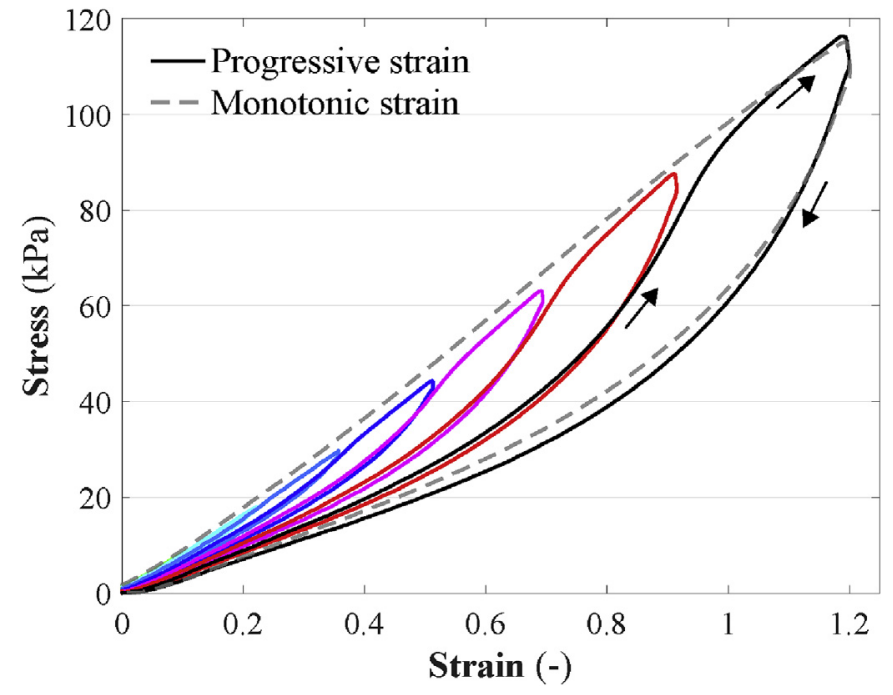

Fig. 4. Representative first loading/unloading stress-strain curves of swollen composite hydrogels at different applied strains. The monotonic loading/unloading (indicated by arrows) up to $70 \%$ deformation of a virgin swollen composite hydrogel is shown in dashed line.

the first cycle $\left(28 \mathrm{~kJ} / \mathrm{m}^{3}\right.$ in 1 st cycle, $5 \mathrm{~kJ} / \mathrm{m}^{3}$ in $\left.2 \mathrm{nd}\right)$ and then remains almost the same. In spite of the aforementioned reduction in energy dissipation, the hysteresis behavior in cyclic loading of the composite hydrogel is still more pronounced compared to the neat hydrogel (Fig. 3-c). As evident in Fig. 3-b, the changes in the hysteresis behavior of the composite hydrogel is accompanied by the stiffness reduction

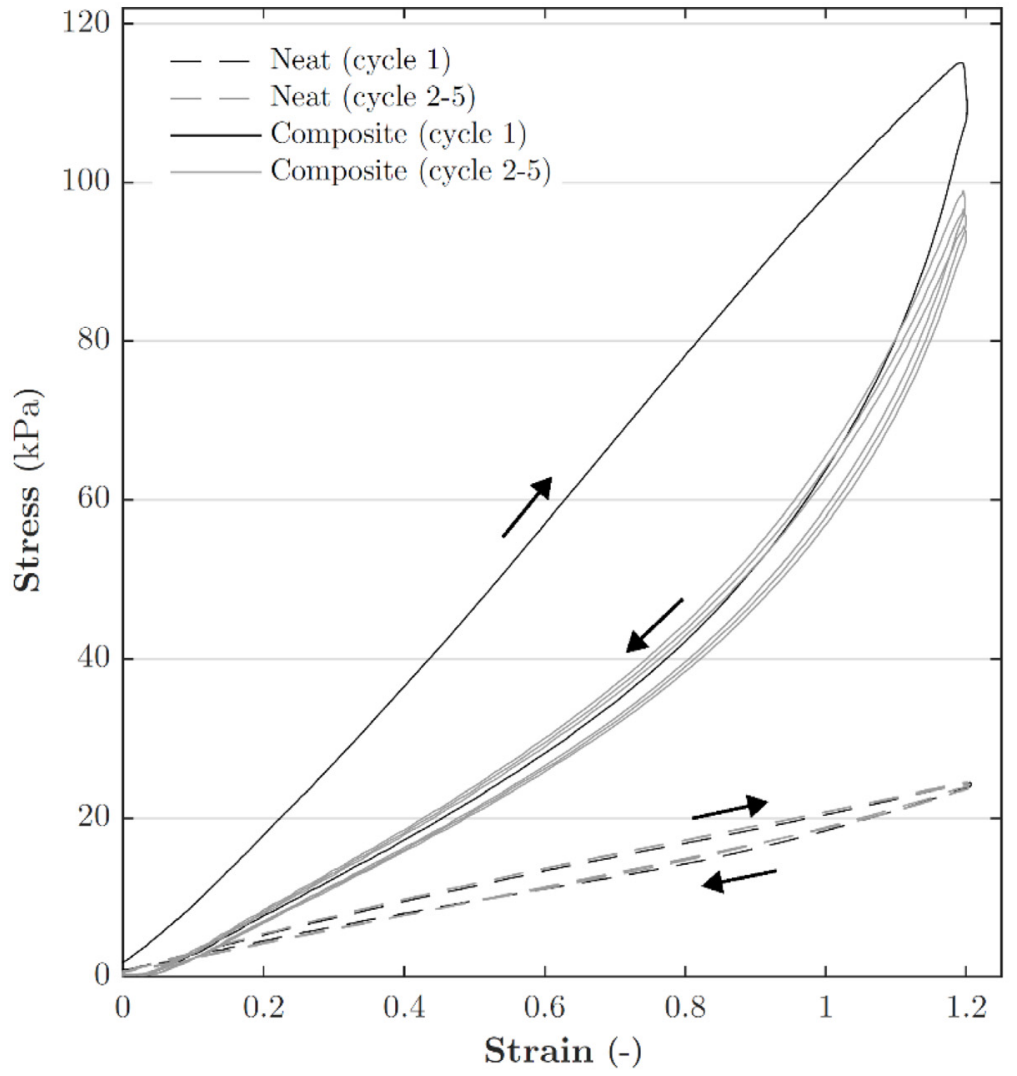

(a)

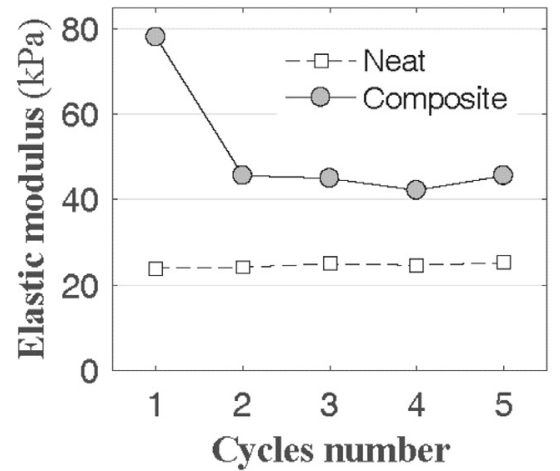

(b)

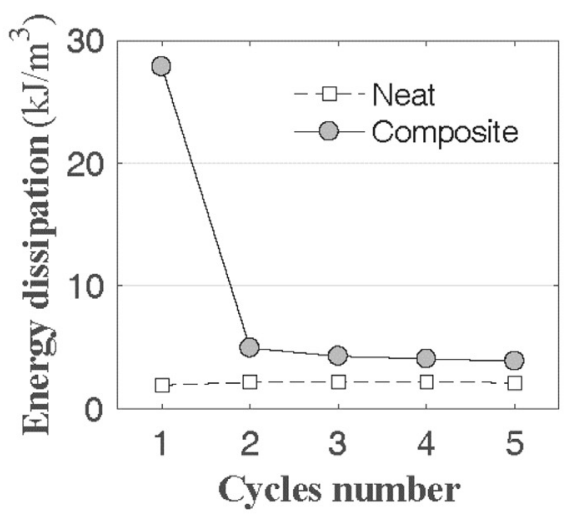

(c)

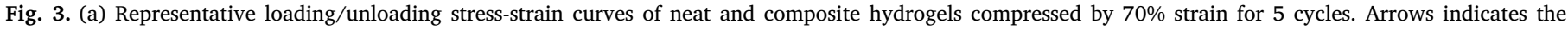

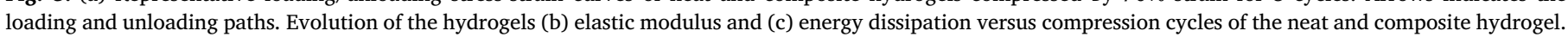




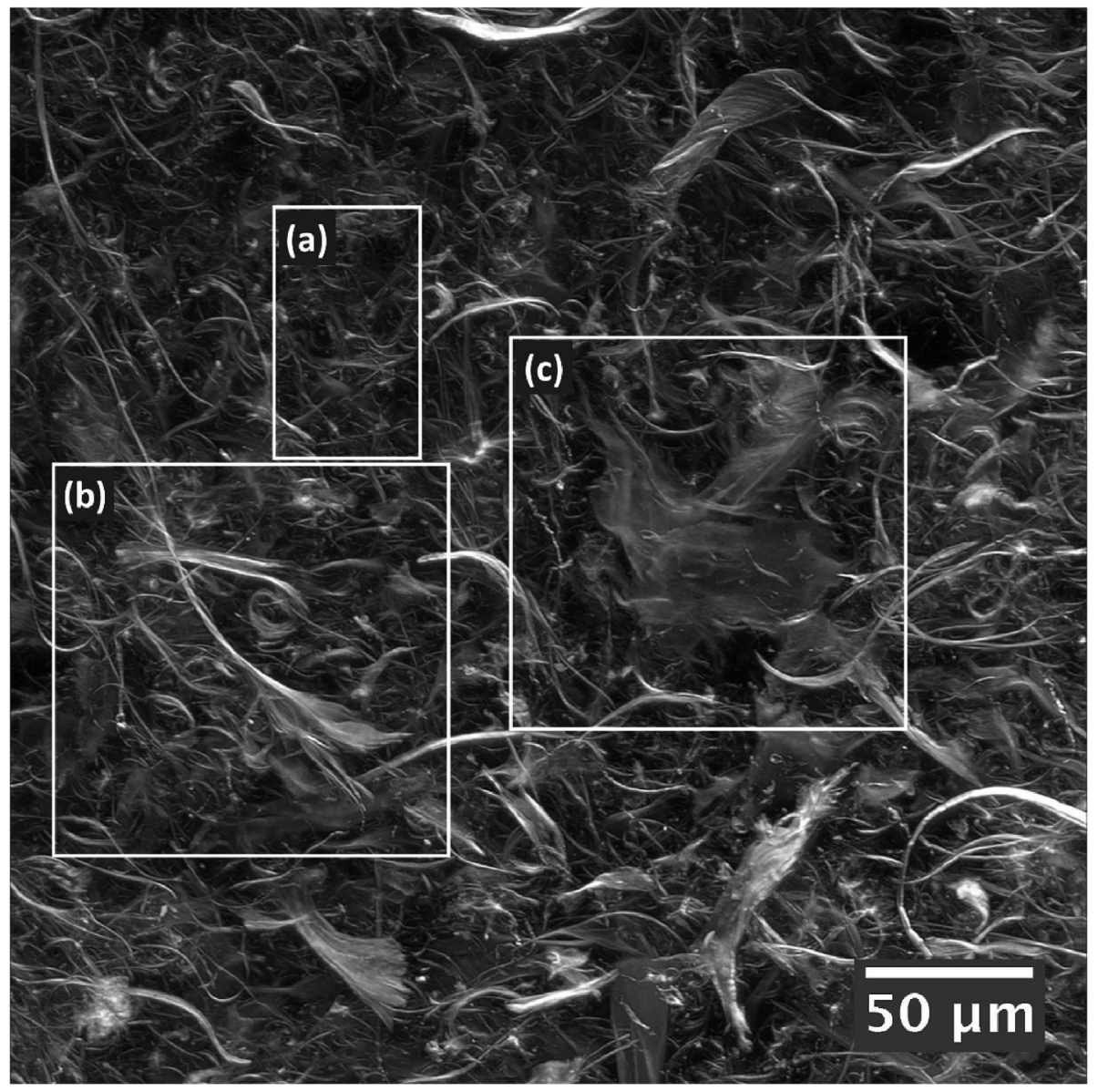

Fig. 5. Z-stack projection of NFC fibers in swollen composite hydrogel showing a large diversity in size and morphology (a) randomly oriented NFC (b) agglomerated NFC in bundles (c) clouds of more dispersed NFC. The corresponding 3D animation of the images is provided in supplementary results.

(a)

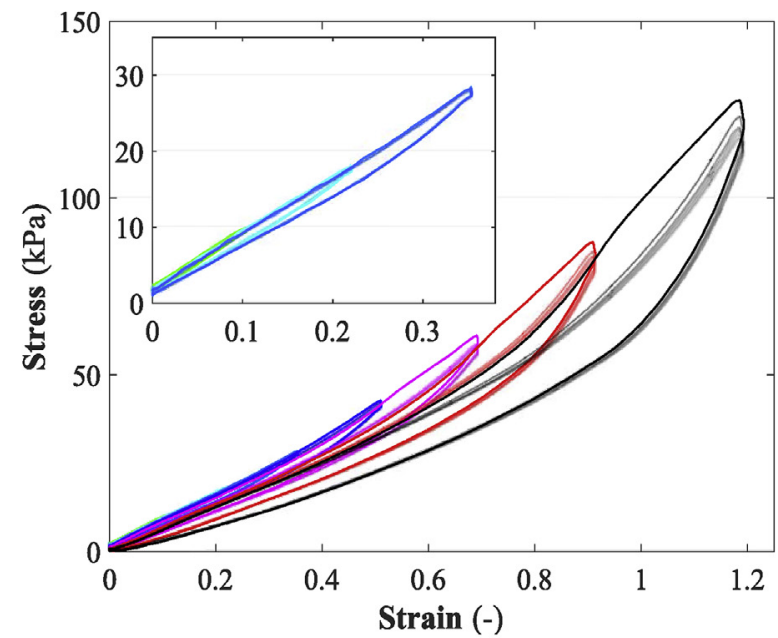

(b)

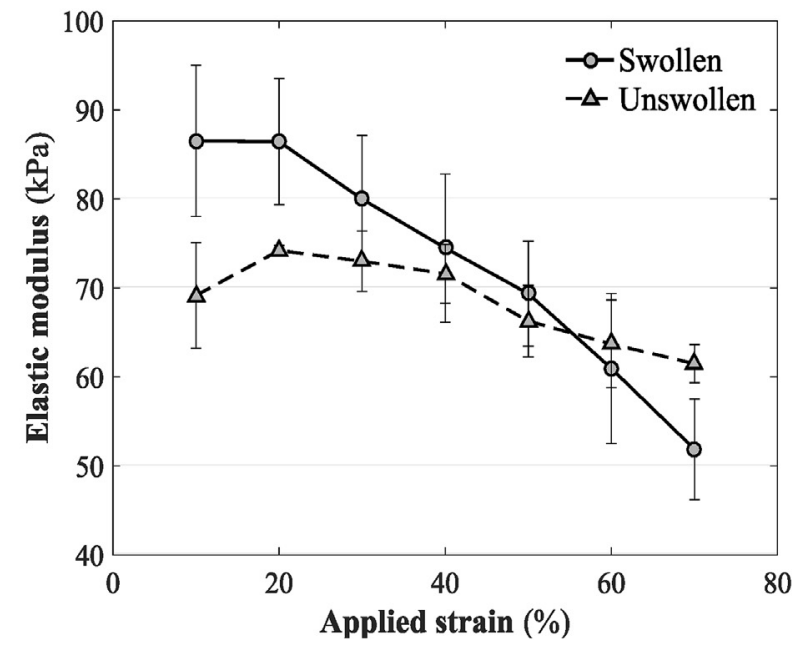

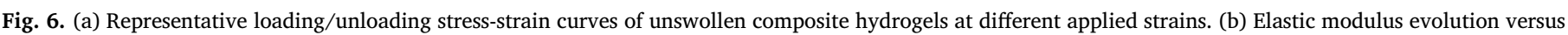
applied strain of swollen (solid line) and unswollen (dashed line) composite hydrogel.

(from $80 \mathrm{kPa}$ to $45 \mathrm{kPa}$ ) in the first cycle. The modulus of the composite hydrogel after reduction is still significantly higher than the neat hydrogel $(25 \mathrm{kPa})$.

The effect of loading level on the hydrogels' mechanical response is investigated by applying successive cycles of different strain amplitudes (ranging from 10 to 70\%). As illustrated in Fig. 4, the loading response of the composite hydrogel is significantly affected by the applied loading history. Each loading curve follows the path of the unloading curve of the previous cycle. When the strain exceeds the maximum amplitude previously applied, the initial loading response is recovered 

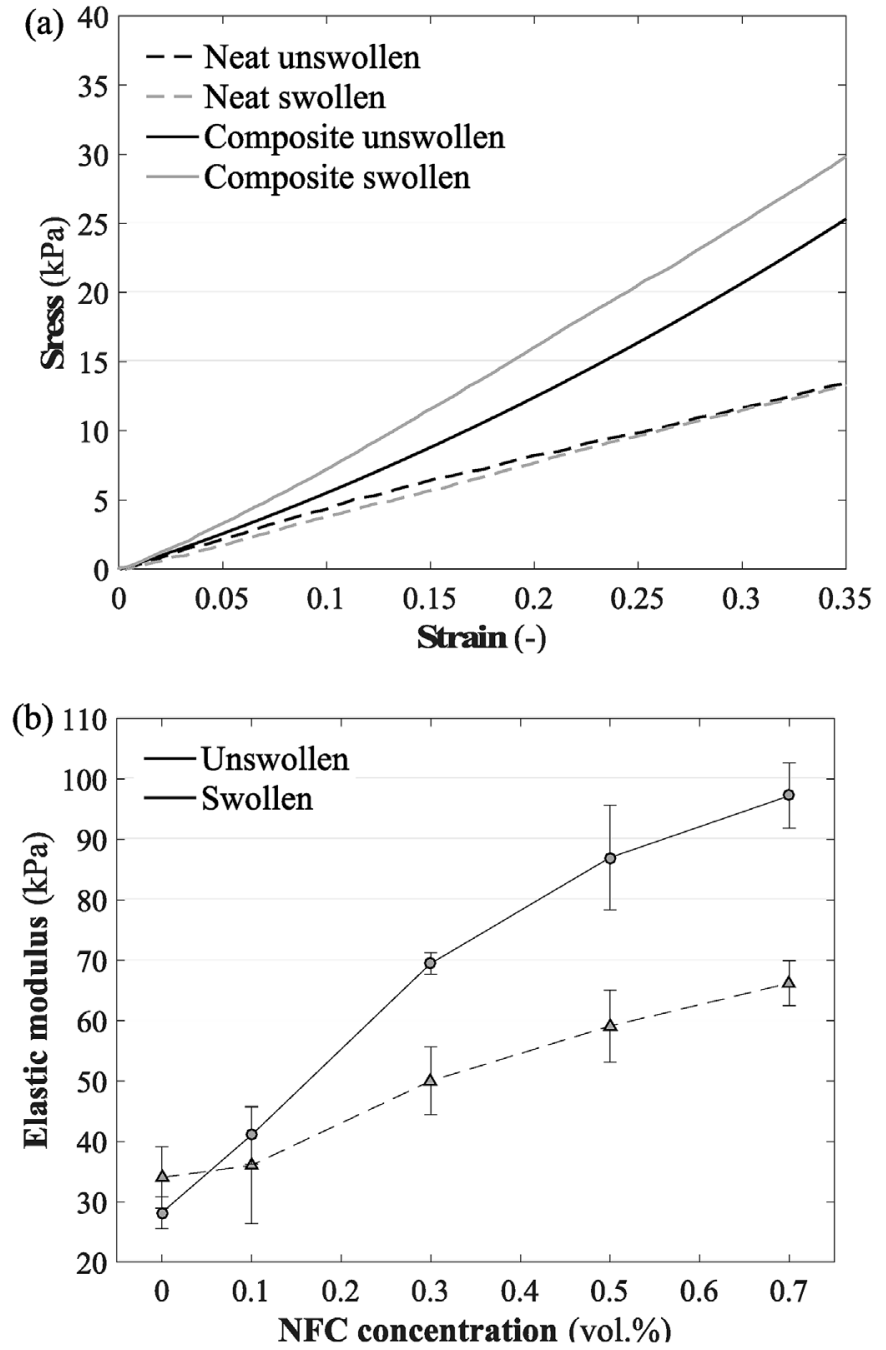

Fig. 7. (a) Representative loading response of neat and composite hydrogel in as-prepared (unswollen) and swollen states. (b) Elastic moduli of neat and composite hydrogels reinforced with different NFC concentration in as-prepared (unswollen) and swollen state.

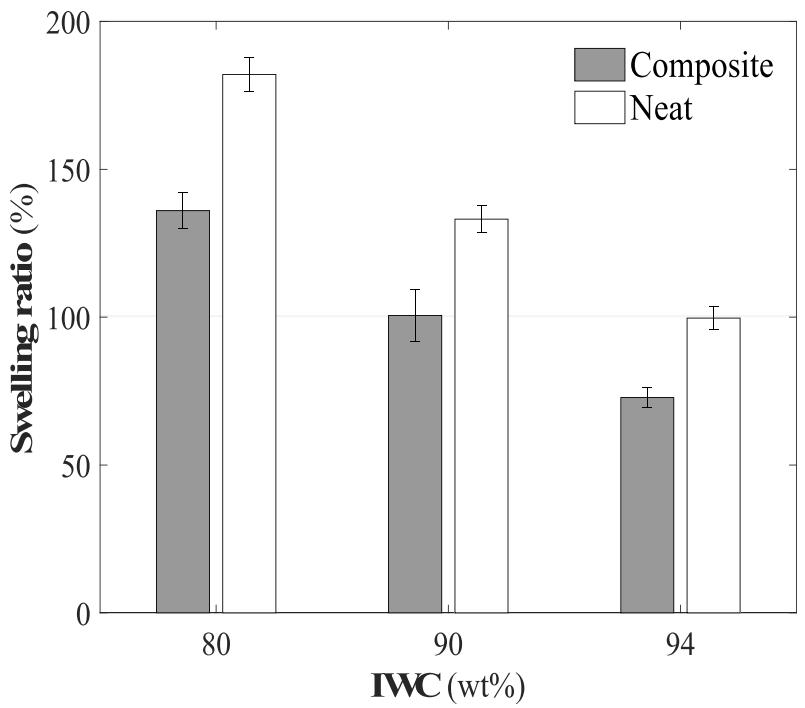

Fig. 8. The swelling ratio of the neat hydrogel with different initial water contents (IWC) that is almost identical to the monotonous stress-strain behavior of an intact sample with no loading history (see Fig. 4 - Dashed line).

Note that a softening behavior similar to the one of the present composite hydrogel (Fig. 4) has been observed in elastomers, mainly filled rubbers which is conventionally denoted as "Mullins effect" [9,30-34]. It is reported that the stiffness reduction due to the Mullins effect can be recovered when the material is subjected to relaxation for a period of time in the order of few hours [34]. Such recovery is not observed for the present composite hydrogel even after 1 week relaxation through immersion in PBS.

Various constitutive relations have been reported in the literature to model Mullin effect. Among them Xuanhe Zhao et al. [35] and Zheming Gao et al. [36] used network alteration theory to describe the softening behavior in interpenetrating polymer networks and nanocomposite hydrogels respectively. It is also shown [37] that such constitutive relations can serve in finite element simulation to predict the hydrogel loading response. Yet, the current available models in the literature do not address the role of swelling and water content on the softening behavior. Moreover, they can not reflect any stiffness recovery process by dehydration-rehydration cycles that can potentially occur in physically crosslinked networks like NFC.

\subsection{Swelling effects}

To investigate the influence of the swelling on softening behavior of the composite hydrogel, the cyclic loading tests is also performed on unswollen, as-prepared composite hydrogels ( $90 \%$ water content). The obtained results shown in Fig. 6 clearly indicate the difference in the softening behavior in cyclic loading response of the as-prepared composite hydrogel compared to the swollen one. In unswollen composite hydrogel no softening behavior is observed when the applied strains are lower than $40 \%$ (see inserted figure in Fig. 6-a). However, when the applied strain is increased to $70 \%$ a stiffness degradation of maximum $14 \%$ is observed (Fig. 6-b).

Comparison of the loading response of the composite hydrogel at the steady swollen state and as-prepared (unswollen) condition indicates a significant increase in the stiffness due to the swelling. In contrast the swelling of the neat hydrogel is associated with a slight decrease in the stiffness (see Fig. 7-a). Furthermore, as shown in Fig. 7-b, the stiffening mechanism due to the swelling in the composite hydrogel becomes more pronounced when the NFC volume fraction is increased.

The rheology results on PEGDM-NFC composite hydrogel precursor [13] showed that composite hydrogel is composed of two networks; a covalently bonded PEGDM network as well as the network of NFC fibers formed through physical entanglements and hydrogen bonds (see Fig. 5). The latter suggests that the stiffening of the composite hydrogel by swelling could also be explained by the following phenomena: (i) The NFC network in the composite hydrogel highly stretches due to the swelling of the matrix. The pre-strained network of elastically active NFC fibers provides more efficient reinforcement effect. (ii) The reinforcement effect of the NFC network reduces the swelling ratio and consequently prevents the hydrogel matrix to achieve its equilibrium swollen state. The latter introduces residual stresses in the polymer network that contributes to the enhancement of the apparent compression elastic modulus.

The softening of the composite hydrogel under cyclic loading can be therefore explained by the degradation of the integrity of highly stretched NFC network in the swollen state. This is similar to what has been observed in cyclic deformation of double polymer network [30,38] as well as semi interpenetrated network hydrogels [9,35,39].

The abovementioned hypothesis regarding the swelling effects on reorientation and disintegration of NFC network under cyclic loading, is examined by tuning the matrix swelling degree. To this end, composite hydrogel with the same NFC concentration $(0.5$ vol.\%) and different initial water contents (IWC $=80-94 \%$ wt.) are evaluated under cyclic deformation. As shown in Fig. 8, using the same molecular weight of 


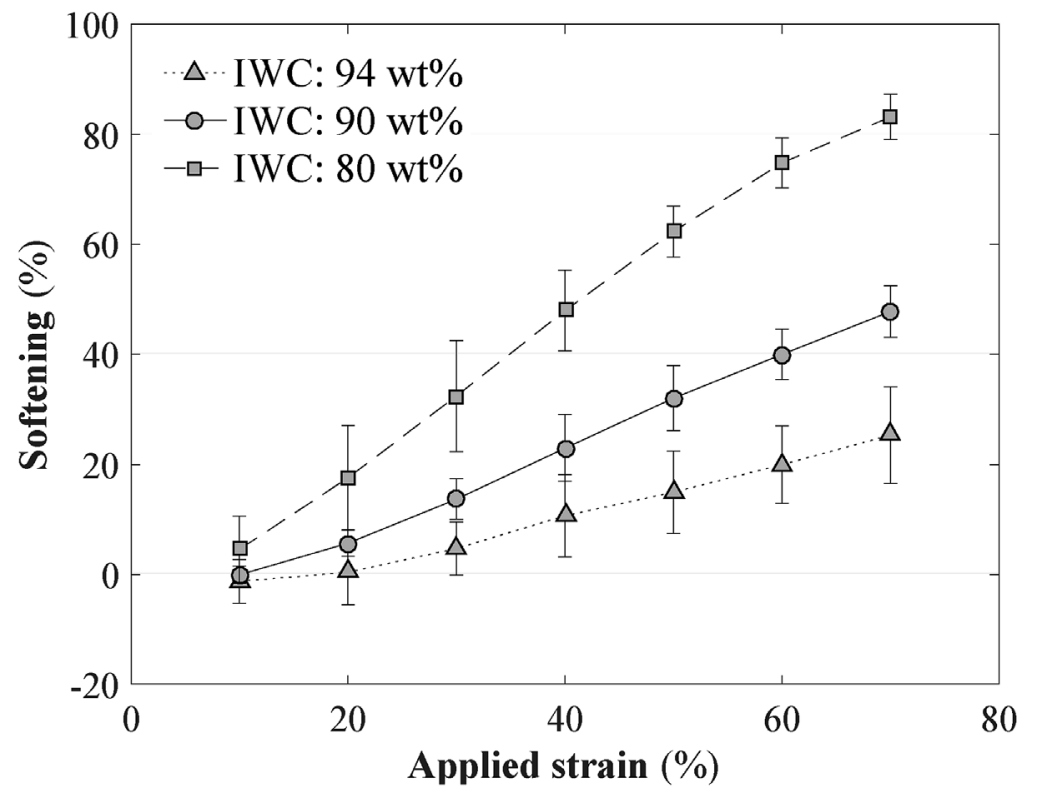

(a)

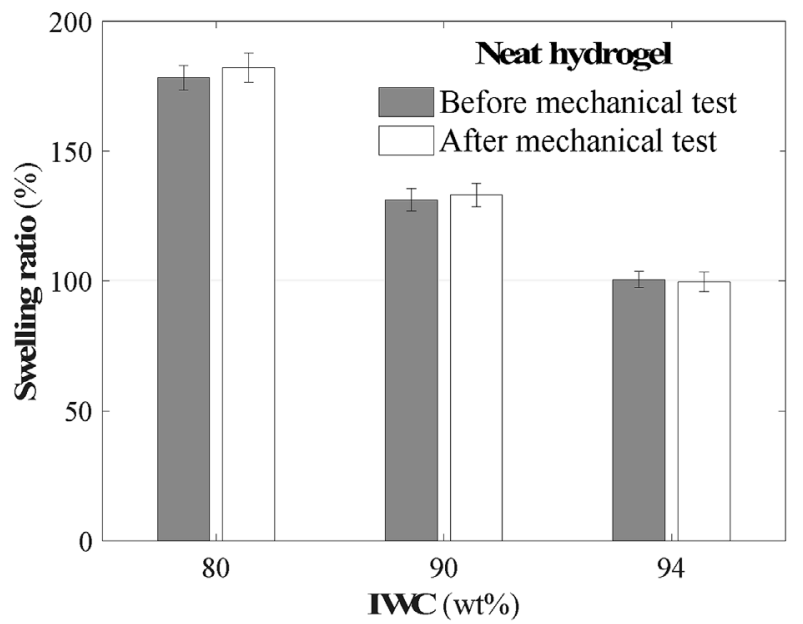

(b)

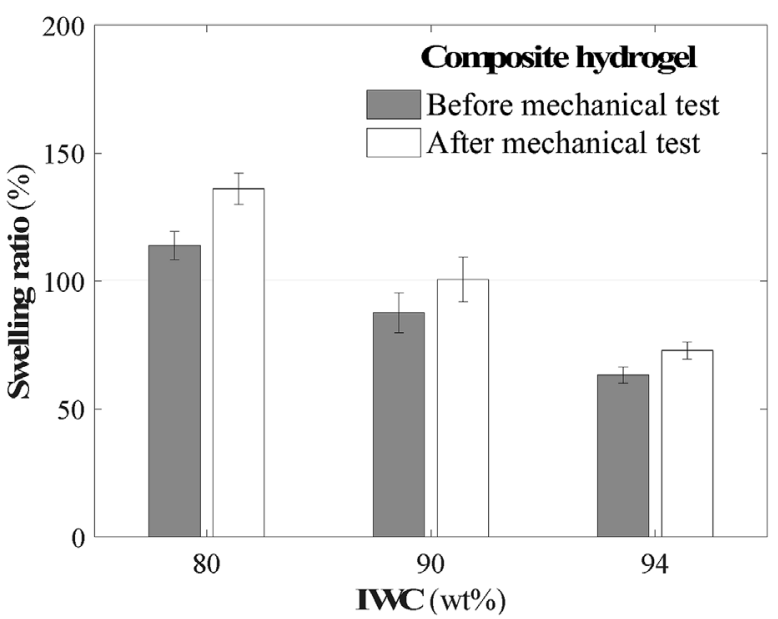

(c)

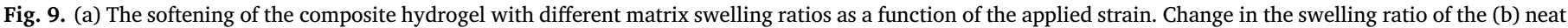
and (c) composite hydrogels after the progressive strain mechanical test.

$20 \mathrm{kDa}$ (i.e. the same ratio between the pre-polymer chain length and the methacrylate crosslink sites), the swelling ratio of the hydrogel can be tuned by altering the IWC.

The softening behavior of the composite hydrogel with different swelling ratios (i.e. different initial water contents) are compared in Fig. 9-a. The results clearly demonstrate that the softening behavior depends on the swelling degree of the hydrogel matrix. Given the fact that no softening is observed in the neat hydrogels, the observed swelling effects on softening behavior are due to the change of NFC network morphology. As shown in Fig. 9-b the disintegration of NFC network leads to an increase in the swelling degree of composite hydrogel under cyclic loading. No change in the swelling ratio is observed in the neat hydrogels after the cyclic loading, similar to the trend in the preceding analyses.

Note that, the initial loading response of composite hydrogel can be recovered by $85 \%$ when the tested sample is completely dried and rehydrated. Such result further confirms that the observed softening behavior under cyclic loading is mainly due to the change in the morphology of the interpenetrated NFC and polymer network rather than a permanent damage to the PEGDM network. The obtained results certainly call for development of more robust material models to take in to account the effect of swelling and hydration on the softening behavior.

\section{Conclusions}

The high cycle fatigue test of poly(ethylene glycol) dimethacrylate (PEGDM) composite hydrogel reinforced with nanofibrillated cellulose (NFC) fiber was successfully performed at physiological condition: maximum $20 \%$ strain. The elastic modulus of the composite hydrogel decreases by almost $10 \%$ mainly over the first loading cycles and then remains constant up to 10 million cycles. Similar tests on the neat hydrogel indicate no stiffness decrease. Low cycle fatigue test shows that the observed softening of the composite hydrogel behave similarly to the Mullins effect. It is associated with a gradual disintegration and 
initial re-arrangement of fiber network. An extensive parametric study incorporating the influence of swelling and the constituent materials suggests that the NFC fibers network is highly stretched in the swollen state and that the extent of the initial softening during cyclic loading is correlated to the swelling degree. Consequently, the softening can be minimized by lowering the extent of the composite hydrogel swelling.

\section{Acknowledgement}

This work was financed by the Swiss National Science Foundation (grant CR 2312-137743). The Applied Wood Materials Laboratory, led by $\mathrm{Dr}$ T. Zimmerman at EMPA Dübendorf, Switzerland, is acknowledged for the support in preparing and providing the NFCs. We thank the groups of Prof. Dominique Pioletti and of Prof. Christopher Moser at EPFL for fruitful discussions, the group of Prof. H. Frauenrath at EPFL, specifically Dr R. Marty for his help in the synthesis of PEGDM and the BIOP team at EPFL for the support on microscopy.

\section{References}

[1] K. Akhilesh, K. Akhilesh, K. Akhilesh, K. Akhilesh, K. Akhilesh, K. Akhilesh, Hydrogels for biomedical applications, Adv. Drug Deliv. Rev. 64 (Supplement) (décembre 2012) 18-23.

[2] A.S. Hoffman, Hydrogels for biomedical applications, Adv. Drug Deliv. Rev. 64 (Supplement) (décembre 2012) 18-23.

[3] H.M. Ávila, et al., Biocompatibility evaluation of densified bacterial nanocellulose hydrogel as an implant material for auricular cartilage regeneration, Appl. Microbiol. Biotechnol. 98 (17) (Sep. 2014) 7423-7435.

[4] T.L. Sun, et al., Physical hydrogels composed of polyampholytes demonstrate high toughness and viscoelasticity, Nat. Mater. 12 (10) (Oct. 2013) 932-937.

[5] D. Shi, Biomedical Devices and Their Applications, Springer Science \& Business Media, 2004.

[6] D.P.M. Brogly, Forces involved in adhesion, in: L.F.M. da Silva, A. Öchsner, R.D. Adams (Eds.), Handbook of Adhesion Technology, Springer Berlin Heidelberg, 2011, pp. 39-63.

[7] A. Schmocker, et al., A photopolymerized composite hydrogel and surgical implanting tool for a nucleus pulposus replacement, Biomaterials 88 (22) (2016) 110-119.

[8] K.T. Nguyen, J.L. West, Photopolymerizable hydrogels for tissue engineering applications, Biomaterials 23 (22) (Nov. 2002) 4307-4314.

[9] J. Djonlagić, D. Žugić, Z. Petrović, High strength thermoresponsive semi-IPN hydrogels reinforced with nanoclays, J. Appl. Polym. Sci. 124 (4) (mai 2012) 3024-3036.

[10] J. Hu, et al., High fracture efficiency and stress concentration phenomenon for microgel-reinforced hydrogels based on double-network principle, Macromolecules 45 (23) (décembre 2012) 9445-9451.

[11] M. Zhong, Y.-T. Liu, X.-M. Xie, Self-healable, super tough graphene oxide-poly (acrylic acid) nanocomposite hydrogels facilitated by dual cross-linking effects through dynamic ionic interactions, J. Mater. Chem. B 3 (19) (2015) 4001-4008.

[12] A.C. Borges, et al., Nanofibrillated cellulose composite hydrogel for the replacement of the nucleus pulposus, Acta Biomater. 7 (9) (Sep. 2011) 3412-3421.

[13] A. Khoushabi, et al., Photo-polymerization, swelling and mechanical properties of cellulose fibre reinforced poly(ethylene glycol) hydrogels, Compos. Sci. Technol. 119 (Nov. 2015) 93-99.

[14] A. Thorvaldsson, J. Silva-Correia, J.M. Oliveira, R.L. Reis, P. Gatenholm, P. Walkenström, Development of nanofiber-reinforced hydrogel scaffolds for nucleus pulposus regeneration by a combination of electrospinning and spraying technique, J. Appl. Polym. Sci. 128 (2) (Apr. 2013) 1158-1163.
[15] K. Kabiri, H. Omidian, M.J. Zohuriaan-Mehr, S. Doroudiani, Superabsorbent hydrogel composites and nanocomposites: a review, Polym. Compos. 32 (2) (Feb. 2011) 277-289.

[16] R. Hartwell, et al., A novel hydrogel-collagen composite improves functionality of an injectable extracellular matrix, Acta Biomater. 7 (8) (Aug. 2011) 3060-3069.

[17] A. Gloria, F. Causa, R.D. Santis, P.A. Netti, L. Ambrosio, Dynamic-mechanical properties of a novel composite intervertebral disc prosthesis, J. Mater. Sci. Mater. Med. 18 (11) (Jul. 2007) 2159-2165.

[18] S.J. Eichhorn, et al., Review: current international research into cellulose nanofibres and nanocomposites, J. Mater. Sci. 45 (1) (Sep. 2009) 1-33.

[19] T. Miyamoto, S. Takahashi, H. Ito, H. Inagaki, Y. Noishiki, Tissue biocompatibility of cellulose and its derivatives, J. Biomed. Mater. Res. A 23 (1) (Jan. 1989) $125-133$.

[20] E. Entcheva, H. Bien, L. Yin, C.-Y. Chung, M. Farrell, Y. Kostov, Functional cardiac cell constructs on cellulose-based scaffolding, Biomaterials 25 (26) (Nov. 2004) 5753-5762.

[21] A. Sannino, C. Demitri, M. Madaghiele, Biodegradable cellulose-based hydrogels: design and applications, Materials 2 (2) (Apr. 2009) 353-373.

[22] A. Schmocker, A. Khoushabi, C. Schizas, P.-E. Bourban, D.P. Pioletti, C. Moser, Miniature probe for the delivery and monitoring of a photopolymerizable material, J. Biomed. Optic. 20 (12) (2015) 127001-127001.

[23] S.C.W. Chan, S.J. Ferguson, B. Gantenbein-Ritter, The effects of dynamic loading on the intervertebral disc, Eur. Spine J. 20 (11) (Nov. 2011) 1796.

[24] Y. Shikinami, Y. Kotani, B.W. Cunningham, K. Abumi, K. Kaneda, A biomimetic artificial disc with improved mechanical properties compared to biological inter vertebral discs, Adv. Funct. Mater. 14 (11) (Nov. 2004) 1039-1046.

[25] P.H. Corkhill, C.J. Hamilton, B.J. Tighe, Synthetic hydrogels VI. Hydrogel composites as wound dressings and implant materials, Biomaterials 10 (1) (Jan. 1989) 3-10.

[26] A. Joshi, et al., Functional compressive mechanics of a PVA/PVP nucleus pulposus replacement, Biomaterials 27 (2) (Jan. 2006) 176-184.

[27] J. Yang, F. Xu, Synergistic reinforcing mechanisms in cellulose nanofibrils composite hydrogels: interfacial dynamics, energy dissipation, and damage resistance, Biomacromolecules 18 (8) (Aug. 2017) 2623-2632.

[28] S. Lin-Gibson, et al., Synthesis and characterization of PEG dimethacrylates and their hydrogels, Biomacromolecules 5 (4) (Jul. 2004) 1280-1287.

[29] S. Josset, P. Orsolini, G. Siqueira, A. Tejado, P. Tingaut, T. Zimmermann, Energy consumption of the nanofibrillation of bleached pulp, wheat straw and recycled newspaper through a grinding process, Nord. Pulp Pap Res. J. 29 (1) (2014) 167-175.

[30] R.E. Webber, C. Creton, H.R. Brown, J.P. Gong, Large strain hysteresis and Mullins effect of tough double-network hydrogels, Macromolecules 40 (8) (avril 2007) 2919-2927.

[31] W. Dong, C. Huang, Y. Wang, Y. Sun, P. Ma, M. Chen, Superior mechanical properties of double-network hydrogels reinforced by carbon nanotubes without organic modification, Int. J. Mol. Sci. 14 (11) (Nov. 2013) 22380-22394.

[32] M.A. Haque, T. Kurokawa, G. Kamita, J.P. Gong, Lamellar bilayers as reversible sacrificial bonds to toughen hydrogel: hysteresis, self-recovery, fatigue resistance, and crack blunting, Macromolecules 44 (22) (Nov. 2011) 8916-8924.

[33] L. Zhang, J. Zhao, J. Zhu, C. He, H. Wang, Anisotropic tough poly(vinyl alcohol) hydrogels, Soft Matter 8 (40) (2012) 10439-10447.

[34] J. Diani, B. Fayolle, P. Gilormini, A review on the Mullins effect, Eur. Polym. J. 45 (3) (Mar. 2009) 601-612.

[35] X. Zhao, A theory for large deformation and damage of interpenetrating polymer networks, J. Mech. Phys. Solid. 60 (2) (février 2012) 319-332.

[36] Q. Wang, Z. Gao, A constitutive model of nanocomposite hydrogels with nanoparticle crosslinkers, J. Mech. Phys. Solid. 94 (Sep. 2016) 127-147.

[37] F. López Jiménez, S. Pellegrino, Constitutive modeling of fiber composites with a soft hyperelastic matrix, Int. J. Solid Struct. 49 (3) (Feb. 2012) 635-647.

[38] J.P. Gong, Why are double network hydrogels so tough? Soft Matter 6 (12) (Jun. 2010) 2583-2590.

[39] S.E. Bakarich, G.C. Pidcock, P. Balding, L. Stevens, P. Calvert, M. in het Panhuis, Recovery from applied strain in interpenetrating polymer network hydrogels with ionic and covalent cross-links, Soft Matter 8 (39) (2012) 9985-9988. 